\title{
EIT Reconstruction Algorithms: Pitfalls, Challenges and Recent Developments
}

\author{
William R.B. Lionheart \\ Department of Mathematics, UMIST, UK
}

\begin{abstract}
We review developments, issues and challenges in Electrical Impedance Tomography (EIT), for the 4th Conference on Biomedical Applications of EIT, Manchester 2003. We focus on the necessity for three dimensional data collection and reconstruction, efficient solution of the forward problem and present and future reconstruction algorithms. We also suggest common pitfalls or "inverse crimes" to avoid.

This paper is dedicated to the memory of the author's Mother, Sheila Breckon, 1926-2003
\end{abstract}

\section{Introduction}

As the papers in this special issue and the 4th Conference on Biomedical Applications of EIT show, reconstruction algorithms for electrical impedance tomography remain an active and exciting area of research. In this article we aim to draw attention to the best current practice as well to pitfalls to avoid, also to highlight some active areas of development and some of the promising methods that have not yet been implemented in practical algorithms. We hope this paper will be a useful contribution for those new to the field as well as being thought provoking for those of us who have been working in the area for some time. Like any review this article has a personal slant on the subject, it also fails to be either a comprehensive review or a history of the subject. In some areas we have deliberately provided less detail where there accessible articles covering the material, in this volume or elsewhere.

By Electrical Impedance Tomography (EIT) we mean the process of estimating internal admittivity (complex conductivity) changes from low frequency current and voltage measurements through a system of electrodes at the surface. Although the details of the instrumentation design varies between biomedical, geophysical and industrial applications the mathematics of the reconstruction problem is essentially the same. The main differences being in the configuration of the electrodes used, and the a priori information available.

\section{Three dimensionality}

In most cases where electrical imaging is used the problem is genuinely threedimensional. An exception being industrial or non-destructive testing problems where the conductivity and the electrodes can be assumed independent of one coordinate. Why then is there such a large body of published work in which data is collected on a three dimensional body and the reconstruction performed assuming the body was two 
dimensional? There are two main reasons: speed and the fact that to some limited extent it worked. The issues of speed include the speed of data collection. The belief that electrodes arranged in a single plane would yield an adequate reconstruction of the conductivity in that plane, and consequent use of a small number of electrodes resulted in a fast data collection system. The number of electrodes applied is also a significant factor when they have to be applied individually to the skin which in itself is a time consuming process. However it is known that data measured on a three dimensional body cannot be fitted accurately to any two dimensional conductivity distribution 41]. Moreover attempts to fit a two dimensional model result in errors of position and shape of anomalies [29, 74]. Another factor in the choice of two dimensional reconstruction methods was the cost of fast processors and memory required to perform three-dimensional forward modelling and reconstruction, which in the 1980s and early 1990s were prohibitive.

Of these factors the one which remains is the inconvenience of applying a fully three dimensional system (for example multiple planes) of electrodes to a human subject, a technological problem which must be overcome if EIT is to be used effectively as a medical imaging technique.

\subsection{Is it tomography?}

It is worth mentioning here that EIT, despite its now traditional name agreed at the first Sheffield EIT meeting in 1986, is not tomographic in that it is not possible to reconstruct an image slice-by-slice. (One can of course reconstruct a three dimensional conductivity distribution and then display it on any desired slice.) The physical explanation for this is that, unlike X-rays, low frequency electric current cannot be confined to a plane even by a system of external electrodes, and that a change in conductivity anywhere in the domain can affect all measurements not just those on a ray path.

An obvious extension of the traditional array of equally spaced electrodes in a plane to a three dimensional data collection system is to employ multiple planes of electrodes. If electrodes are excited in pairs on a given plane it is necessary to make measurements of voltage on the electrodes in other planes as well. If one is modifying the traditional planar system for this purpose, and voltages are measured between adjacent pairs of electrodes, one must also make measurements between planes as well for a complete set of transfer impedance data. Clearly only one measurement between one pair of electrodes on adjacent planes is sufficient by superposition. This configuration is however only an expedient way to employ inflexible equipment and is not likely to be an ideal data collection scheme. Optimal current drive patterns have been described for a cylindrical tank with several planes of electrodes [31. The optimal arrangement of electrodes for imaging brain or lung function for example has yet to be determined.

There is interesting connection between EIT and tomographic imaging which has yet to be exploited in a practical reconstruction algorithm. Suppose that one is able to measure the complete transfer impedance on a plane intersecting a three dimensional object, while there is no known way of reconstructing the conductivity on this plane Greenleaf and Uhlmann [33] show that the integral of the conductivity over that plane is known. Suppose now that we use a large number of surface electrodes so that the transfer impedance, and hence the integral of the conductivity, is known for a wide class of planes. Reconstructing the conductivity reduces to the problem of inverting 
a Radon plane transform with limited data.

\section{Forward Problem solution}

For accurate EIT reconstruction it a prerequisite to have a model capable of predicting the voltages on electrodes for a given conductivity distribution. The conductivity is adjusted until the voltages fit to measurement precision. This forward model must also be capable of predicting the electric fields in the interior given the conductivity, as this is used in the reconstruction algorithms (See section [5.2). It is clear that the voltages on the electrodes must be predicted to a precision better than the accuracy of the measurements. But the accuracy required of the interior electric fields and consequently the sensitivity of measurements to conductivity changes, is a matter which has received little attention.

\subsection{Choice of method}

As we are interested in non-homogeneous conductivity distributions on irregular domains Finite Element Method (FEM) is the natural choice [81, 11, 70, 73, 74, 56, 55, [52], although finite difference [54] and finite volume methods [23] 84 have also been employed. Where the conductivity is known and homogeneous in some sub domain, especially a neighbourhood of the boundary, and attractive proposition is to use a hybrid boundary element and finite element method [36. As the highest field strengths are typically near the edges of electrodes this is where the densest mesh is required. With the Boundary Element Method (BEM) only the surface needs to be discretized with a consequent saving on the size of matrix to be inverted. However BEM alone can only be used on homogeneous regions, and is useful for fitting impedance values to known regions [21] . On the other hand the matrix for the hybrid system has a dense blocks for the BEM and for the coupling between BEM and FEM, and care has to be taken to use an efficient solution scheme for such a matrix.

\subsection{Custom versus commercial FEM code}

For medical applications, as well as industrial applications where conductive electrodes are in contact with an aqueous solution, the Complete Electrode Model must be employed for accurate prediction of the electrode voltages [65]. This takes account of both the shunting effect of the conducting electrodes and the contact impedance layer between the electrode and the solution (see (9) below). As this is not a standard type of boundary condition it is not easily implemented in commercial finite element packages where the source code is not accessible. This together with the necessity to update the system matrix rapidly with a revised conductivity militates against the use of a closed source commercial FEM solver. Another factor is the ease with which the Jacobian can be assembled from low level access to products of gradients of shape functions (see section [5.2). Even FEMLAB [19] which has considerable low-level access to internal structures through Matlab does not allow this at present.

In practice the assembly of the FEM system matrix, at least for simple linear tetrahedral elements, is easily implemented and the step from two to three dimensions requires only the calculations of integrals of products of shape functions over faces under electrodes [74, 56]. The steps which involve considerably more time and care are mesh generation and the efficient solution of the linear system. The design of 
three dimensional mesh generators is a major research topic in itself, and we have yet to find an existing program, commercial or free, which is ideal for EIT. The main requirements are for an efficient mesh of an object composed of smooth but irregular surfaces, which respects interior boundaries, and electrodes on the surface. The mesh density needs to be determined as a function of position so that high field strengths (for example near electrodes) can be accurately represented without excessive density in areas where the field varies slowly. Even if such a program were available one would still need to measure the external shape of the body accurately, and in the case of the human head intricate internal structures such as the skull needs to be segmented from X-Ray CT or MRI scans [5].

\subsection{Efficient forward solution}

The computational complexity of the three-dimensional forward problem means that attention has to be paid to the efficiency of the forward solution. For two dimensional problems a standard approach to solving the linear system resulting from the finite element discretization is to employ Cholesky factorization (or $L U$ decomposition for the case of complex admittivity) together with forward and backward substitution to give the potential for each applied current pattern 32]. As the system matrix is sparse, renumbering the degrees of freedom to reduce the fill in of non-zeros in the Cholesky factors is common practice. The Symmetric Multiple Minimum Degree algorithm is a standard choice which tends to move degrees of freedom with higher "valencies" (coupled to many others) to the bottom of the vector. With the complete electrode model degrees of freedom include both finite element nodal values and electrode voltages. The Matlab function symmmd implements the multiple minimum degree algorithm, and for the complex case the Column Multiple minimum degree colmmd function can be used which reduces the fill in for the $L U$ factorization. It needs to be stressed that although node renumbering can be expensive it needs to be done only once for each mesh and the result stored for future use. In three dimensions iterative solution methods have become more attractive, although each iterative step must be applied to the multiple right-hand sides for each current pattern. Iterative methods also have an advantage that for when the update to the conductivity is small the change in the potential will also be small. This means that the number of iterations need to solve the forward solution can be reduced as the iteration can be initialized with the previously computed values.

For the real case Conjugate Gradient method is a favourite choice. Convergence is improved using a preconditioner which is an approximate inverse to the system matrix. If the conductivity does not vary over too wide a range during the reconstruction process this approximate inverse can be precomputed based on a typical conductivity, and as the inversion is typically performed by incomplete Cholesky factorization the same consideration of node renumbering applies as for direct methods. For the complex case other Krylov subspace iterative methods [57] such as GMRES can be used [58].

\subsection{Accuracy of forward solution}

In iterative methods time can be saved if a solution is not required to full machine precision, and as our boundary voltage data is not so accurate it is unnecessary to solve to this accuracy. The accuracy required for the interior electric fields and hence the Jacobian is not well understood. That said the accuracy of the finite element 
method approximation is well studied and there are a priori estimates [3] for the error in terms of the mesh size ( $r$ convergence) and the order of the elements used ( $h$ convergence). There are also a posteriori error estimates based on calculated solutions 3] which have not yet been widely used in EIT. Although there is some work using higher order elements 74 the best choice of element for EIT remains an open problem, and the possibility of using vector elements [10] to calculate electric fields and current densities accurately in the interior is largely unexplored for our problem. The use of infinite elements to model unbounded regions, or at least regions which while bounded have a substantial part where we have no surface data, is an interesting possibility [75]. Possible applications include limbs when the torso is being imaged, as well as problems where the body is treated as an infinite half space such as in geophysical imaging or the use of a small surface electrode array in medical EIT.

\subsection{Multigrid and adaptive meshing}

So far we have considered the situation in which the same mesh is used throughout the forward solution process. One attractive alternative is to solve first on a course grid to give the crude features of a solution which is then extrapolated to a finer grid where a mode accurate solution is calculated. A systematic treatment of this cycling between finer and courser grids is used in multigrid solution algorithms. At least for regular grids the theoretical optimum of solving a system with time complexity $O(N)$ can be approached for $N$ degrees of freedom. The geometric problems of calculating a hierarchy of grids for a complex object can be avoided using algebraic multigrid methods which are described elsewhere in this special issue 64.

Another strategy for reducing the solution time is to adaptively vary the finite element mesh used. During the forward solution process for a fixed conductivity the mesh density is increased where the high field strengths are found and decreased where the potential is gently varying. This results in a more accurate solution than a regular mesh with the same number of degrees of freedom. One complexity for our problem is that we have multiple right-hand sides, so we can choose either to use the same mesh for all current patterns, in which case it may be unnecessarily fine near passive electrodes, or to use a different mesh for each drive configuration. Although typically the highest field strengths appear near the edges of electrodes (including passive electrodes if the contact impedance is low), sharp contrasts in conductivity can also give rise to high field strengths. For example an aperture in the skull (low conductivity) for a relatively high conductivity blood vessel or nerve. An adaptive meshing algorithm will increase the mesh density here once the conductivity contrast has been predicted by the reconstruction algorithm. The thesis of Mollinari [52] explores the use of adaptive meshing in both forward and inverse problems in EIT and gives a clear indication that this is a fruitful area for further study.

\section{Regularised Newton Methods}

While numerous ad hoc reconstruction methods have been tried for EIT the standard approach is to use one of a family of regularized Newton-type methods. The approach is to some extent the obvious one: the problem is non-linear so linearize, the linear problem is ill-posed so regularize, the linear approximation cannot reconstruct large contrasts or complex geometries so the process must be applied iteratively. There are of course many variations on this basic approach and we will sketch some typical ones. 
First let us assume that the conductivity $\sigma$ has been represented by a finite number of parameters s. In the simplest case, this is taken as a sum of basis functions such as the characteristic functions of a set of regular or irregular voxels, or smooth basis functions. Other choices would include a detailed model involving conductivity values as well as parameters describing shape internal regions [38, 39.

\subsection{Regularization}

Our forward operator $F$ gives us $\mathbf{V}=F(\mathbf{s})$ the simulated voltages at the boundary. We will leave aside the adaptive current approach [28, 12] where the current patterns used depend on the estimated conductivity. As the goal is to fit the voltage measurements $\mathbf{V}_{\text {meas }}$, the simplest approach is to minimize the sum of squares error

$$
\left\|\mathbf{V}_{\text {meas }}-F(\mathbf{s})\right\|^{2}
$$

the so called output least squares approach. Here $\|\cdot\|$ is the standard 2-norm on vectors. In practice it is not usual to use the raw least squares approach, but at least a weighted sum of squares which reflects the reliability of each voltage. Such approaches are common both in optimization and the statistical approach to inverse problems.

Minimization of the voltage error (for simple parameterizations of $\sigma$ ) is doomed to failure as the problem is illposed [68. In practice the minimum lies in a long narrow valley of the objective function[11]. For a unique solution one must include additional information about the conductivity, an example is to include a penalty $G(\mathbf{s})$ for highly oscillatory conductivites, hence in our problem we seek to minimize

$$
f(\mathbf{s})=\left\|\mathbf{V}_{\text {meas }}-F(\mathbf{s})\right\|^{2}+G(\mathbf{s}) .
$$

A typical simple choice [70] is

$$
G(\mathbf{s})=\alpha^{2}\left\|L\left(\mathbf{s}-\mathbf{s}_{\mathrm{ref}}\right)\right\|^{2}
$$

where $L$ is a matrix approximation to some partial differential operator and $\mathbf{s}_{\text {ref }}$ a reference conductivity (for example including known anatomical features). The minimisation of $f$ represents a trade-off between fitting the data exactly and not making the derivatives of $\sigma$ too large, the trade off being controlled by the regularization parameter $\alpha$. Other smooth choices of $G$ include the inverse of a Gaussian smoothing filter [9]. In these cases where $G$ is smooth and for $\alpha$ large enough the Hessian of $f$ will be positive definite, we can then deduce that $f$ is a convex function [77, $\mathrm{Ch} 2$ ], so that a critical point will be a strict local minimum, guaranteeing the success of smooth optimization methods. Such regularization however will prevent us from reconstructing conductivities with a sharp transition, such as an organ boundary. Including the Total Variation, that is the integral of $|\nabla \sigma|$, still rules out wild fluctuations in conductivity while allowing step changes. The cost is that the inclusion of an absolute value destroys the differentiability of $f$ and means that we must employ non-smooth optimization methods. This is both more difficult and computationally expensive than smooth optimization problems. Early applications to EIT 22] employed total variation regularization to the linearized EIT problem. A detailed review of the literature on this subject as well as a more efficient optimization algorithm can be found in the thesis [7]. See [78] for analysis of Total Variation regularization of EIT.

In the statistical approach [77, $\mathrm{Ch} 4$ ] to regularization the minimizer of $f$ maximizes the a posteriori probability (the MAP estimate) assuming independent 
Gaussian error with mean zero and unit variance on the measurements and the $a$ priori information on $\sigma$ represented by the probability distribution $\exp (-G(\mathbf{s}) / 2)$. To many the probabilistic approach to regularization provides a more rational frame work for the recovery of a finite number of parameters from a discrete set of data than an approach to regularization based on functional analysis. Ideally we would incorporate a probability model for the errors in the data and using a prior probability density for the unknown parameters find not just the maximum of the posterior probability density, but a more compete description of the probability of a range of conductivity images. For an excellent review of the statistical regularization in EIT see [37. Despite the low spatial resolution of EIT the temporal resolution is high. In biomedical applications to time varying conductivity distributions, caused for example by blood or air flow, images are correlated temporally as well as spatially. Statistical time series methods such can be used to include both spatial and temporal regularization [72, 76]. Applications to industrial process modelling [61] are better developed than biomedical applications, although this is a highly active area of research.

\subsection{Linearized Problem}

Consider the simplified case where $F(\mathbf{s})$ is replaced by a linear approximation

$$
F\left(\mathbf{s}_{0}\right)+J\left(\mathbf{s}-\mathbf{s}_{0}\right)
$$

where $J$ is the Jacobian matrix of $F$ calculated at some initial conductivity estimate $\mathbf{s}_{0}$ (not necessarily the same as $\left.\mathbf{S}_{\mathrm{ref}}\right)$. The function to be minimized (2) with regularizing penalty term (3) becomes a quadratic function when $F$ is replaced by its linear approximation (4). Defining $\delta \mathbf{s}=\mathbf{s}-\mathbf{s}_{0}$ and $\delta \mathbf{V}=\mathbf{V}_{\text {meas }}-F\left(\mathbf{s}_{0}\right)$ the solution to the linearized regularization problem is given by

$$
\delta \mathbf{s}=\left(J^{*} J+\alpha^{2} L^{*} L\right)^{-1}\left(J^{*} \delta \mathbf{V}+\alpha^{2} L^{*} L\left(\mathbf{s}_{\mathrm{ref}}-\mathbf{s}_{0}\right)\right)
$$

(where $J^{*}$ is the conjugate transpose of $J$ ) or any of the equivalent forms [67]. While there are many other forms of regularization possible for a linear ill-conditioned problem this generalized Tikhonov (or Tikhonov-Phillips) regularization has the benefit that the a priori information it incorporates is made explicit and that under Gaussian assumptions it is the statistically defensible MAP estimate. If only a linearised solution is to be used with a fixed initial estimate $\mathbf{s}_{0}$ the Jacobian $J$ and a factorization of $\left(J^{*} J++\alpha^{2} L^{*} L\right)$ can be precalculated off-line. The efficiency of this calculation is then immaterial and the regularized solution can be calculated using the factorization with complexity $O\left(N^{2}\right)$ for $N$ degrees of freedom in the conductivity (which should be smaller than the number of independent measurements). Although $L U$ factorization would be one alternative, perhaps a better choice is to use the Generalized Singular Value Decomposition GSVD [34, which allows the regularized solution to be calculated efficiently for any value of $\alpha$. The GSVD is now a standard tool for understanding the effect of the choice of the regularization matrix $L$ in a linear ill-conditioned problem, and has been applied to linearised EIT 9 . The use of a single linearized Tikhonov regularized solution is widespread in medical industrial and geophysical EIT, the NOSER algorithm [17] being a well known example. Such algorithms one step linear algorithms were the first three-dimensional algorithms to be applied to experimental data from tanks [30, and the human thorax [50, 51]. It must be emphasized that a linearized solution will only be accurate when the true conductivity is close to the initial estimate. 


\subsection{Backprojection}

It is an interesting historical observation that in the medical and industrial applications of EIT numerous authors have calculated $J$ and then proceeded to use ad hoc regularized inversion methods to calculate an approximate solution. Often these are variations on standard iterative methods which, if continued would for a well posed problem converge to the Moore-Penrose generalised solution. It is a standard method in inverse problems to use an iterative method but stop short of convergence (Morozov's stopping criteria tells us to stop when the output error first falls below the measurement noise). Many linear iterative schemes can be represented as a filter on the singular values [77, Ch 1]. However they have the weakness that the a priori information included is not as explicit as in Tikhonov regularization. One extreme example of the use of an ad hoc method is the method described by Kotre [40] in which the normalized transpose of the Jacobian is applied to the voltage difference data. In the Radon transform used in X-Ray CT [53], the formal adjoint of the Radon transform is called the back projection operator. It produces at a point in the domain the sum of all the values measured along rays through that point. Although not an inverse to the Radon transform itself, a smooth image can be obtained by backprojecting smoothed data, or equivalently by back-projecting and then smoothing the resulting image.

The Tikhonov regularization formula (5) can be interpreted in a loose way as the back-projection operator $J^{*}$ followed be the application of the spatial filter $\left(J^{*} J++\alpha^{2} L^{*} L\right)^{-1}$. Although this approach is quite different from the filtered back projection along equipotential lines of Barber and Brown [4, 59] it is sometimes confused with this in the literature. Kotre's back projection was until recently widely used in the process tomography community for both resistivity (ERT) and permittivity (ECT) imaging [80]. Often supported by the fallacious arguments, in particular that it is fast (it is no faster than the application of any precomputed regularized inverse) and that it is commonly used (only by those who know no better). In an interesting development the application of a normalised adjoint to the residual voltage error for the linearised problem was suggested for ECT, and later recognised as yet another reinvention of the well known Landweber iterative method [79]. Although there is no good reason to use pure linear iteration schemes directly on problems with such small a number of parameters as they can be applied much faster using the SVD, an interesting variation is to use such a slowly converging linear solution together with projection on to a constraint set. A method which has been shown to work well in ECT [15.

\subsection{Iterative solutions}

The use of linear approximation is only valid for small deviations from the reference conductivity. In medical problems conductivity contrasts can be large, but there is a good case for using the linearized method to calculate a change in admittivity between two states, measured either at different times or with different frequencies. Although this has been called "dynamic imaging" in EIT the term difference imaging is now preferred (dynamic imaging is a better used to describe statistical time series method such as 69]). In industrial ECT modest variations of permittivity are commonplace. In industrial problems and in phantom tanks it is possible to measure a reference data set using a homogenious tank. This can be used to calibrate the forward model, in particular the contact impedance can be estimated [35. In an in vivo measurement 
there is no such possibility and it may be that the mismatch between the measured data and the predictions from the forward model is dominated by the errors in electrode position, boundary shape and contact impedance rather than interior conductivity. Until these problems are overcome it is unlikely, in the author's opinion, to be worth using iterative non-linear methods in vivo using individual surface electrodes. Note however that such methods are in routine use in geophysical problems [82, 44, 45]. Computational complexity of both forward solution and inversion of the linearized system meant that, although iterative nonlinear algorithms had been implemented for simulated data on modest meshes earlier [43] it was only in the mid 1990s that affordable computers had sufficient floating point speed and memory to handle sufficiently dense three-dimensional meshes to fit tank data adequately [71, 74]

The essence of non-linear solution methods is to repeat the process of calculating the Jacobian and solving a regularised linear approximation. However a common way to explain this is to start with the problem of minimizing $f$, which for a well chosen $G$ will have a critical point which is the minimum. At this minimum $\nabla f(\mathbf{s})=\mathbf{0}$ which is a system of $N$ equations in $N$ unknowns which can be solved by multi-variable NewtonRaphson method. In practice for noisy data there may not be an exact solution. The Gauss-Newton approximation to this, which neglects terms involving second derivatives of $F$, is a familiar Tikhonov formula updating the $n$th approximation to the conductivity parameters $\mathbf{s}_{n}$

$$
\mathbf{s}_{n+1}=\mathbf{s}_{n}+\left(J_{n}^{*} J_{n}+\alpha^{2} L^{*} L\right)^{-1}\left(J_{n}^{*}\left(\mathbf{V}_{\text {meas }}-F\left(\mathbf{s}_{n}\right)\right)+\alpha^{2} L^{*} L\left(\mathbf{s}_{\text {ref }}-\mathbf{s}_{n}\right)(6)\right.
$$

where $J_{n}$ is the Jacobian evaluated at $\mathbf{s}_{n}$, and care has to be taken with signs . Notice that in this formula the Tikhonov parameter is held constant throughout the iterations, by contrast Levenberg-Marquardt [6] method applied to $\nabla f=0$ would add a diagonal matrix $\lambda D$ in addition to the regularization term $\alpha^{2} L^{*} L$ but would reduce $\lambda$ to zero as a solution was approached. For an interpretation of $\lambda$ as a Lagrangian multiplier for an optimization constrained by a trust region see [77, Ch 3]. Another variation on this family of methods is, given an update direction from the Tikhonov formula, to do an approximate line search to minimize $f$ in that direction. Both methods are described in [77, Ch 3].

The parameterization of the conductivity can be much more specific than voxel values or coefficients of smooth basis functions. One example is to assume that the conductivity is piecewise constant on smooth domains and reconstruct the shapes parameterized by Fourier series [38, 39] or by level sets 25. For this and other model based approaches the same family of smooth optimization techniques can be used as for simpler parameterizations, although the Jacobian calculation may be more involved.

\section{Jacobian calculations}

In optimization based methods it is often necessary to calculate the derivative of the voltage measurements with respect to a conductivity parameter. The complete matrix of partial derivatives of voltages with respect to conductivity parameters is the Jacobian matrix, sometimes in the medical and industrial EIT literature called the sensitivity matrix, or the rows are called sensitivity maps. We will describe here the basic method for calculating this efficiently with a minimal number of forward solutions. Let it be said first that there are methods where the derivative is calculated only once, although the forward solution is calculated repeatedly as the conductivity is updated. This is the difference between Newton-Kantorovich method and Newton's 
method. There are also Quasi-Newton methods in which the Jacobian is updated approximately from the forward solutions that have been made. Indeed this has been used in geophysics [45]. It also worth pointing out that were the conductivity is parameterized in a nonlinear way for example using shapes of an anatomical model, the Jacobian with respect to those new parameters can be calculated using the chain rule.

\subsection{Dissipated power}

The derivation of the Jacobian is best understood in terms of the dissipated power. We take for simplicity a real conductivity $\sigma$. The potential $u$ satisfies

$$
\nabla \cdot \sigma \nabla u=0
$$

Using the weak form of (7) or using Green's identity, for any $w$

$$
\int_{\Omega} \sigma \nabla u \cdot \nabla w d V=\int_{\partial \Omega} w \sigma \frac{\partial u}{\partial n} d S
$$

Here $d V$ and $d S$ are volume and surface measures, $n$ the outward normal vector and $\Omega$ the domain (the body). We use the complete electrode model 65 with contact impedance $z_{l}$ which says that on electrode $E_{l}$

$$
u=V_{l}-z_{l} \sigma \frac{\partial u}{\partial n}
$$

for a constant electrode voltage $V_{l}$, the total current on electrode $E_{l}$ is

$$
\int_{E_{l}} \sigma \frac{\partial u}{\partial n} d S=I_{l}
$$

and $\partial u / \partial n=0$ away from electrodes. For the special case $w=u$ we have the power conservation formula,

$$
\int_{\Omega} \sigma|\nabla u|^{2} d V=\int_{\partial \Omega} u \sigma \frac{\partial u}{\partial n} d S=\sum_{l} \int_{E_{l}}\left(V_{l}-z_{l} \sigma \frac{\partial u}{\partial n}\right) \sigma \frac{\partial u}{\partial n} d S
$$

hence

$$
\int_{\Omega} \sigma|\nabla u|^{2} d V+\sum_{l} \int_{E_{l}} z_{l}\left(\sigma \frac{\partial u}{\partial n}\right)^{2}=\sum_{l} V_{l} I_{l}
$$

This simply states that the power input is dissipated either in the domain $\Omega$ or by the contact impedance layer under the electrodes.

\subsection{Standard formula for Jacobian}

We now take perturbations $\sigma \rightarrow \sigma+\delta \sigma, u \rightarrow u+\delta u$ and $V_{l} \rightarrow V_{l}+\delta V_{l}$, with the current in each electrode $I_{l}$ held constant. We calculate the first order perturbation, and argue as in [16 that the terms we have neglected are higher order in the $L^{\infty}$ norm on $\delta \sigma$. The details of the calculation are given for the complete electrode model case in [55], the result is

$$
\sum_{l} I_{l} \delta V_{l}=-\int_{\Omega} \delta \sigma|\nabla u|^{2} d V
$$

This gives only the total change in power, to get the change in voltage on a particular electrode $E_{m}$ when a current pattern is driven in some or all of the other 
electrodes we simply solve for the special 'measurement current pattern' $\widetilde{I}_{l}^{m}=\delta_{l m}$. To emphasize the dependance of the potential on a vector of electrode currents $\mathbf{I}=\left(I_{1}, \ldots, I_{L}\right)$ we write $u(\mathbf{I})$. The hypothetical measurement potential is $u\left(\mathbf{I}^{m}\right)$, by contrast the potential for the $d$-th drive pattern is $u\left(\mathbf{I}^{d}\right)$. Applying the power perturbation formula (13) to $u\left(\mathbf{I}^{d}\right)+u\left(\mathbf{I}^{m}\right)$ and $u\left(\mathbf{I}^{d}\right)-u\left(\mathbf{I}^{m}\right)$ and then subtracting gives the familiar formula

$$
\delta V_{d m}=-\int_{\Omega} \delta \sigma \nabla u\left(\mathbf{I}^{d}\right) \cdot \nabla u\left(\mathbf{I}^{m}\right) d V
$$

Standard arguments based on series expansions of operators [16] 11] can be used to show that this is indeed the Fréchet derivative for $\delta \sigma \in L^{\infty}(\Omega)$, considerable care is needed to show that the voltage data is Fréchet differentiable in other normed spaces, such as those needed to show that the total variation regularization scheme works [78. For a finite dimensional subspace of $L^{\infty}(\Omega)$ a proof of differentiability is given by [37.

In the special case of the Sheffield adjacent pair drive, adjacent pair measurement protocol, commonly used in two dimensional EIT we have potentials $u_{i}$ for the $i$ th drive pair and voltage measurement $V_{i j}$ for a fixed current $I$ a across the $j$-th measurement pair

$$
\delta V_{i j}=-\frac{1}{I^{2}} \int_{\Omega} \delta \sigma \nabla u_{i} \cdot \nabla u_{j} d V
$$

To calculate the Jacobian matrix one must choose a discretizarion of the conductivity. The simplest case is to take the conductivity to be piecewise constant on polyhedral domains such as voxels or tetrahedral elements. Taking $\delta \sigma$ to be the characteristic function of the $k$-th voxel we have for a fixed current pattern

$$
\frac{\partial V_{d m}}{\partial \sigma_{k}}=-\int_{\text {voxel k }} \nabla u\left(\mathbf{I}^{d}\right) \cdot \nabla u\left(\mathbf{I}^{m}\right) d V
$$

With the double indices $d m$ renumbered as a single index, these functions form the elements of the Jacobian matrix $J$. For the case of a complex admittance one must repeat this calculation taking care to use the real component of dissipated power $V_{l} \bar{I}_{l}$. Some EIT and capacitance tomography systems use a constant voltage source and in this case the change in power of an increase in admittivity will have the opposite sign to the constant current case.

Some iterative nonlinear reconstruction algorithms, such as nonlinear Landweber, or non-linear conjugate gradient [77, 67] require the evaluation of transpose (or adjoint in the complex case) of the Jacobian multiplied by a vector $J^{*} z$. For problems where the Jacobian is very large it may be undesirable to store the Jacobian and then apply its transpose to $z$. Instead the block of $z_{i}$ corresponding to the $i$ th current drive is written as a distributed source on the measurement electrodes. A forward solution is performed with this as the boundary current pattern so that when this measurement field is combined with the field for the drive pattern as (16), and this is accumulated to give $J^{*} z$. For details of this applied to diffuse optical tomography see [2], and for a general theory of adjoint sources see [77. For an example of application to EIT see for example [56, Ch 4] and to electromagnetic imaging [24].

For fast calculation of the Jacobian using (16) one can precompute the integrals of products of finite element basis functions over elements. If non constant basis functions are used on elements, or higher order elements used one could calculate the product of gradients of FE basis functions at quadrature points in each element. As this depends only on the geometry of the mesh and not on the conductivity this can be 
precomputed unless one is using an adaptive meshing strategy. The same data is used in assembling the FE system matrix efficiently when the conductivity has changed but not the geometry. It is these factors particularly which make current commercial FEM software unsuitable for use in an efficient EIT solver. While there are some details of efficient methods for Jacobian calculation in the literature (37), more specific implementation details can often be found in theses, such as [81, 11, 70, 74, 56].

\section{Inverse crimes and common pitfalls}

The ill-posed nature of inverse problems means that any reconstruction algorithm will have limitations on what images it can accurately reconstruct and that the images degrade with noise in the data. When developing a reconstruction algorithm it is usual to test it initially on simulated data. Moreover the reconstruction algorithms typically incorporates a forward solver. A natural first test is to use the same forward model to generate simulated data with no simulated noise and to then find to one's delight that the simulated conductivity can be recovered fairly well, the only difficulties being if it violates the a priori assumptions built into the reconstruction and the limitations of floating point arithmetic. Failure of this basic test is used as a diagnostic procedure for the program.

\subsection{Use a different mesh}

If one is fortunate enough to have a good data collection system and phantom, and someone skilled enough to make some accurate measurements with the system one could then progress to attempting to reconstruct images from experimental data. However more often the next stage is to test further with simulated data and it at this stage that one must take care not to cheat and commit a so called "inverse crime" 18, p133]. The best practice is to use an independent forward model, at the very least in the case of a finite element forward model one would use a much finer mesh, and one which was not a strict refinement of the mesh used in the forward solver in the reconstruction. It is an obvious point perhaps but the simulated conductivity must be represented on this mesh and unless we are explicitly using a priori information about for example the location of the boundary of an anomaly the mesh used for simulate data should not be 'known' to the reconstruction program.

\subsection{Simulating noise}

It is necessary to study the effect of measurement error on the reconstruction. The first reflex of the mathematically trained is to use a pseudo random number generator to add independent Gaussian noise to each measurement with an identical variance. There are some tightly controlled laboratory situations where experimentalists can carefully calibrate their apparatus to remove systematic error leaving only random error from discretization of measurements and random effects such as thermal noise. By averaging large numbers of measurements the Central Limit Theorem means that independent identical Gaussian noise is a good approximation to the true statistics of the data error. However in electrical imaging we are rarely in this fortunate situation. Even using phantom tanks there are many sources of error which are hard to calibrate away. The statistical characterisation of instrumentation error in EIT is a topic which requires considerable further study. In vivo studies suffer further sources of error 
including variable contact impedance, motion artifact and variable surface geometry all of which produce correlated errors in the data.

Even simple simulation of discretization error requires some understanding of the measurement system one has in mind. While the data is discretized into a binary representation of the voltage with a fixed precision by an analogue to digital converter (ADC), the input to the ADC has already been scaled by an amplifier. In a Sheffieldtype adjacent pair drive system 13 this scale factor is determined by the position of the measurement pair relative to the drive pair to make best use of the range of the ADC. Multiple drive systems employ different strategies [20, 83]. One should at least add identically distributed noise to suitably scaled measurements. Whatever scheme is chosen for simulating noise it should be carefully described, often a phrase such as " $5 \%$ random noise was added" is used without saying if this is $5 \%$ of the largest voltage (which could completely destroy smaller measurements) or that percentage of each measurement.

\subsection{Pseudo random numbers}

Two small caveats about the use of pseudo random number generators, such as the randn function in Matlab. First that unless the seed for the pseudo-random number generator is changed the same sequence of random numbers is generated each time Matlab is started. It possible therefore that many of us are using the same sequence of "random" numbers! Secondly that for each error level one should generate a sequence or ensemble of pseudo-random errors and perform the reconstruction for each. One can then find the mean error in reconstruction and the mean error in fitting the data, as well as their variance. As the EIT reconstruction problem is nonlinear adding Gaussian error to the data will not produce even multivariate Gaussian reconstruction error, so strictly one would have to consider the full distribution of the errors, but for small noise levels one can expect a linear approximation to be valid. In practice the danger is that the pseudo-random sequence will produce an out-lier, and one plots reconstruction error against data error one would notice the discrepancy, which presumably explains why the culprits usually get away with the "inverse misdemeanour" of not using an ensemble of pseudo random errors.

\subsection{Thou shallst not tweak}

Finally there is some sharp practice which applies equally to simulated and experimental results. The first of these we will call "tweaking". Reconstruction programs have a number of adjustable parameters such as Tikhonov factors and stopping criteria for iteration, as well as levels of smoothing, basis constraints and small variations on algorithms. While there are rational ways of choosing reconstruction parameters based on the data (such as generalized cross validation and L-curve), and on an estimate of the data error (Morotzov's stopping criterion), a practical procedure often employed is to simulate, or measure experimentally, a variety of conductivity distributions the find by trial and error parameters in the reconstruction program which do reasonably well, knowing what the image was meant to be. One could compare this with the training set used for a neural net. We would then not be surprised to find that conductivity distributions close to the training set can be reconstructed satisfactorily. A more interesting test is to see how the algorithm performs when we deviate from the training set. There are plenty of examples of 
conference papers where reconstruction algorithms are shown to perform very well on single circular anomalies, while reconstructions of complex objects with varying contrasts are absent. As we know the problem is illposed, it is inevitable that there are some conductivity distributions which can not be reconstructed well with a particular algorithm, in particular ones which violate the a priori assumptions. It is therefore no dishonour to present the failures as well as the successes. To avoid the temptation to tweak the algorithm to produce the best results given that the correct result is known, the best procedure would be to conduct blind trials for both simulated and experimental data.

The author is aware that what is suggested here best practice is a high standard to aim for, and that examples of this crimes and misdemeanours can be found in his own work, but the intention is to elevate the standard in published work in EIT reconstruction generally and to highlight these pitfalls. While in conference presentations it is acceptable to describe work in progress, and to confess to any inverse crimes and misdemeanours, it is the author's opinion that they should be avoided in journal publications.

\section{Further developments in reconstruction}

In this breif review there is no space to describe in any detail many of the exciting current development in reconstruction algorithms. Fortunately many of them are treated in other articles in this special issue. Before highlighting some of these developments it is worth emphasising that for ill-posed problem a priori information is essential for stable reconstruction algorithms, and it is better that this information is incorporated in the algorithm in a systematic and transparent way. Another general priciple of inverse problems is to think carefully what information is required by the end user. Rather than attempting to produce an accurate image what is often required in medical (and indeed most other) applications is an estimate of a much smaller number of parameters which can be used for diagnosis. For example we may know that a patient has two lungs as well as other anatomical features but we might want to estimate their water content to diagnose pulminary oedema. A sensible strategy would be to devise an anatomical model of the thorax and fit a few parameters of shape and conductivity rather than pixel conductivity values. The disadvantage of this approach is that each application of EIT gives rise to its own specialised reconstruction method, which must be carefully designed for the purpose. In the author's opinion the future development of EIT systems, including electrode arrays and data acquisition systems as well as reconstruction software, should focus increasingly on specific applications, although of course such systems will share many common components.

\subsection{Beyond Tikhonov regularization}

We have also discussed the use of more general regularization functionals including total variation. For smooth $G$ traditional smooth optimization techniques can be used, whereas for total variation the Primal Dual Interior Point Method is promising [7]. In general there is a trade-off between incorporating accurate a priori information and speed of reconstruction. Where the regularization term is a partial differential operator, the solution of the linearized problem is a compact perturbation of a partial differential equation. This suggests that multigrid methods may be used in the solution

of the inverse problem as well. For a single linearized step this has been done for 
the EIT problem by McCormick and Wade [48, and for the non-linear problem by Borcea [6]. In the same vein adaptive meshing can be used for the inverse problem [52]. In both cases there is the interesting possibility to explore the interaction between the meshes used for forward and inverse solution.

At the extreme end of this spectrum we would like to describe the prior probability distribution and for a known distribution of measurement noise and calculate the entire posterior distribution. Rather than giving one image, such as the MAP estimate, this gives a complete description of the probability of any image. If the probability is bimodal for example, one could present the two local maximum probability images. If one needed a diagnosis, say of a tumour, the posterior probability distribution could be used to calculate the probability that a tumour like feature was there. The computational complexity of calculating the posterior distribution for all but the simplest distributions is enormous, however the posterior distribution can be explored using the Markov Chain Monte Carlo Method (MCMC) [37. This was applied to simulated EIT data [26], and more recently to tank data, including in this special issue 49. For this to be a viable technique for the 3D problem highly efficient forward solution will be required, and an efficient and fast 3D MCMC algorithm for EIT presents a serious but very worthwhile challenge.

\subsection{Direct nonlinear methods}

Iterative methods which use optimization methods to solve a regularized problem are necessarily time consuming. The forward problem must be solved repeatedly and the calculation of an updated conductivity is also expensive. The first direct method to be proposed was the Layer Stripping algorithm [63] however this is yet to be shown to work well on noisy data. An exciting recent development is the implementation of a Scattering Transform algorithm proposed by Nachman. Siltanen 62 showed that this can be implemented stably and applied to tank data. The main limitation of this technique is that is inherently two dimensional and no one has found a way to extend it to three dimensions, also in contrast to the more explicit forms of regularization it is not clear what a priori information is incorporated in this method as the smoothing is applied by filtering the data. A strength of the method is its ability to accurately predict absolute conductivity levels. In some cases where long electrodes can be used and the conductivity varies slowly in the direction in which the electrodes are oriented a two dimensional reconstruction may be a useful approximation. This is perhaps more so in industrial problems such as monitoring flow in pipes with ECT or ERT. In some situations a direct solution for a two dimensional approximation could be used as a starting point for an iterative three dimensional algorithm.

Two further direct methods show considerable promise for specific applications. The monotonicity method of Tamburrino et al [66] relies on the monotonicity of the map $\rho \mapsto R_{\rho}$ where $\rho$ is the real resistivity and $R_{\rho}$ the transfer impedance matrix. This method, which is extremely fast, relies on the resistivity of the body to be known to be one of two values. It works equally well in two and three dimensions and is robust in the presence of noise. The time complexity scales linearly with the number of voxels (which can be any shape) and scales cubically in the number of electrodes. It works for purely real or imaginary admittivity, (ERT or ECT), and for Magnetic Induction Tomography for real conductivity. It is not known if it can be applied to the complex case and it requires the voltage measurements on current carrying electrodes.

Linear sampling methods [14, 60] have a similar time complexity and advantages 
as the monotonicity method. While still applied to piecewise constant conductivities, linear sampling methods can handle any number of discrete conductivity values provided the anomalies are separated from each other by the background. The method does not give an indication of the conductivity level but rather locates the jump discontinuities in conductivity. Both monotonicity and linear sampling methods are likely to find application in situations where a small anomaly is to be detected and located, for example breast tumours.

Finally a challenge remains to recover anisotropic conductivity which arises in applications from fibrous or stratified media (such as muscle), flow of non-spherical particles (such as red blood cells), or from compression (for example in soil). The inverse conductivity problem at low frequency is known to suffer from insufficiency of data, but with sufficient prior knowledge (for example [42]) the uniqueness of solution can be restored. One has to take care that the imposition of a finite element mesh does not predetermine which of the family of consistent solutions is found [1].

\section{Conclusions}

In conclusion, until medical EIT data is reconstructed using the best available methods the results will be inconclusive. Many an experimental study has be spoilt when carefully collected data has been attacked by a crude two-dimensional linear reconstruction algorithm and the resulting 'blurry blobs' taken as evidence that EIT is not suitable for the desired task. Careful consideration of a priori information, measurement error and the model parameters required is needed, together with close collaboration between mathematicians and experimentalists. Periodically a mood arises at an EIT meeting that the technique will never find real application in medicine, however it is my contention that a particular application should not be dismissed as impossible until both hardware and software specialists working together have given it their 'best shot'.

\section{Acknowledgements}

The author would like to thank the referees for their helpful comments, and the colleagues, especially Nick Polydorides, who commented on earlier drafts.

\section{References}

[1] Abscal JFP, 2003, The Anisotropic Inverse Conductivity Problem, MSc Thesis, University of Manchester, UK.

[2] Arridge S, 1999, Optical tomography in medical imaging, Inverse Problems, 15, R41-93.

[3] Babuska I, and Strouboulis T, 2001, The Finite Element Method and its Reliability, Oxford University Press, Oxford

[4] Barber D and Brown B, 1986, Recent developments in applied potential tomography-APT, Information Processing in Medical Imaging, ed S L Bacharach, Nijhoff, Amsterdam 106-121.

[5] Bayford RH, Gibson A, Tizzard A, Tidswell AT, and Holder DS, 2001, Solving the forward problem for the human head using IDEAS (Integrated Design Engineering Analysis Software) a finite element modelling tool, Physiol Meas, 22, 55-63.

[6] Borcea L, 2001, A nonlinear multigrid for imaging electrical conductivity and permittivity at low frequency, Inverse Problems, 17, 329-359.

[7] Borsic A, 2002, Regularization Methods for Imaging from Electrical Measurements, PhD thesis, Oxford Brookes University.

[8] Borsic A, McLeod CN and Lionheart WRB, 2001, Total variation regularisation in EIT reconstruction 2nd World Congr. on Industrial Process Tomography (Hannover), 579 -587. 
[9] Borsic A, Lionheart WRB, McLeod CN, 2002, Generation of anisotropic-smoothness regularization filters for EIT, IEEE Trans Med Imaging, 21, 596-603.

[10] Bossavit A, 1998, Computational Electromagnetism, Variational Formulations, Edge Elements, Complementarity, Academic Press, Boston.

[11] Breckon WR, 1990, Image Reconstruction in Electrical Impedance Tomography, PhD Thesis, Oxford Polytechnic.

[12] Breckon WR and Pidcock MK, 1988, Data errors and reconstruction algorithms in electrical impedance tomography Clin Phys Physiol Meas, 9, Suppl A, 105-109.

[13] Brown BH and Seagar AD, 1987, The Sheffield data collection system Clin Phys, Physiol Meas, 8, Suppl A 91-7.

[14] Brühl M, 2001, Explicit Characterization of Inclusions in Electrical Impedance Tomography, SIAM J Math Anal, 32, 1327-1341.

[15] Byars M, 2001, Developments in Electrical Capacitance Tomography, Proc. World Congress on Industrial Process Tomography, Hannover, 542-549.

[16] Calderón AP, 1980, On an inverse boundary value problem, in Seminar on Numerical Analysis and Its Applications to Continuum Physics, 67-73, Rio de Janeiro, Sociedade Brasileira de Matematica.

[17] Cheney, M, Isaacson D, Newell JC, Simske S and Goble J, 1990, NOSER: An algorithm for solving the inverse conductivity problem, Int. J. Imaging Systems \& Technology 2, 66-75.

[18] Colton D, Kress R, 1998, Inverse Acoustic and Electromagnetic Scattering Theory, 2nd edition, Springer, Berlin.

[19] COMSOL, 2000, The FEMLAB reference manual, COMSOL AB, Stockholm.

[20] Cook RD, Saulnier GJ, Gisser DG, Goble JC, Newell JC and Isaacson D., 1994, ACT 3: A high speed high precision electrical impedance tomograph. IEEE Trans. Biomed. Eng. 41, 713-722.

[21] de Munck, JC, Faes, TJC, Heethaar, RM, 2000, The boundary element method in the forward and inverse problem of electrical impedance tomography, IEEE Trans Bio-Med Eng, 47, 792800.

[22] Dobson DC and Santosa F, 1994, An Image Enhancement Technique for Electrical Impedance Tomography, Inverse Problems, 10, 317-334.

[23] Dong, GY, Endo H, Hayano S, Gao SK and Saito Y, 2003, GVSPM for reconstruction in electrical impedance tomography, IEEE Trans. Magnetics, 39, 1630-1633.

[24] Dorn O, Bertete-Aguirre H, Berryman JG, and Papanicolaou GC, 1999, A nonlinear inversion method for 3D electromagnetic imaging using adjoint fields, Inverse Problems, 15, 1523-1558.

[25] Dorn O, Miller EL, Rappaport CM, 2000, A shape reconstruction method for electromagnetic tomography using adjoint fields and level sets, Inverse Problems, 16, 1119-1156

[26] Nicholls GK and Fox C, Prior modelling and posterior sampling in impedance imaging, in Bayesian Inference for Inverse Problems, Proc. SPIE 3459, 116-127, 1998

[27] Gilbert, JR, Moler C, and Schreiber R, 1992 Sparse Matrices in MATLAB: Design and Implementation, SIAM Journal on Matrix Analysis and Applications, 13, 333-356.

[28] Gisser G, Isaacson D and Newell JC, 1987, Current topics in impedance imaging, Clin. Phys. Physiol. Meas., 8 Suppl A, 39-46.

[29] Goble J, 1990, The three-dimensional inverse problem in electric current computed tomography, PhD thesis, Rensselaer Polytechnic Institute, NY, USA.

[30] Goble J and Isaacson D, 1990, Fast reconstruction algorithms for three-dimensional electrical impedance tomography, Proc. IEEE-EMBS Conf, 12(1) 100-101.

[31] Goble J and Isaacson D, 1988, Optimal current patterns for three-dimensional electric current computed tomography, Proc IEEE-EMBS Conf, 11:463-464.

[32] Golub GH and Van Loan CF, 1996, Matrix Computations, 3rd ed, Johns Hopkins University Press, Baltimore.

[33] Greenleaf A and Uhlmann G, 2001, Local uniqueness for the Dirichlet-to-Neumann map via the two-plane transform, Duke Math J, 108, 599-617.

[34] Hansen PC, 1998, Rank-deficient and discrete ill-posed problems: numerical aspects of linear inversion, SIAM, Philadelphia.

[35] Heikkinen LM, Vilhunen T, West RM and Vauhkonen M, 2002, Simultaneous reconstruction of electrode contact impedances and internal electrical properties: II. Laboratory experiments Meas. Sci. Technol., 13, 1855-1861

[36] Hsiao GC, Schnack E, Wendland WL, 2000 Hybrid Coupled Finite Boundary Element Methods for Elliptic Systems of Second Order, Technical report 2000/5, Department of Mathematical Sciences, University of Delaware.

[37] Kaipio J, Kolehmainen V, Somersalo E and Vauhkonen M, 2000,Statistical inversion and Monte Carlo sampling methods in electrical impedance tomography, Inverse Problems 16 1487-1522. 
[38] Kolehmainen V, Arridge SR, Lionheart WRB, Vauhkonen M and Kaipio JP, 1999, Recovery of region boundaries of piecewise constant coefficients of elliptic PDE from boundary data, Inverse Problems 15, 1375-1391.

[39] Kolehmainen V, Vauhkonen M, Kaipio JP and Arridge SR, 2000, Recovery of piecewise constant coefficients in optical diffusion tomography, Optics Express, 7, 468-480.

[40] Kotre CJ, 1989, A sensitivity coefficient method for the reconstruction of electrical impedance tomograms Clin Phys Physiol Meas, 10, 275-281.

[41] Lionheart WRB, 1999, Uniqueness, shape, and dimension in EIT, Annals New York Acad Sci, 873, 466-471.

[42] Lionheart WRB, 1997, Conformal uniqueness results in anisotropic electrical impedance imaging. Inverse Problems, 13, 125-134.

[43] Liu WP, Hua P and Webster JG, 1988, Three-dimensional reconstruction in electrical impedance tomography, Clin Phys Physiol Meas, 9, 131-135

[44] Loke MH and Barker R, 1996, Rapid least-squares inversion of apparent resistivity pseudosections by a quasi-Newton method, Geophysical Prospecting, 44, 131-152.

[45] Loke MH and Barker RD, 1996, Practical techniques for 3D resistivity surveys and data inversion, Geophysical Prospecting, 44, 499-523.

[46] Marquardt D, 1963, An algorithm for least squares estimation of nonlinear parameters, SIAM J Appl Math, 11, 431-441.

[47] Mayavi, 2003, The MayaVi Data Visualizer, http://mayavi.sourceforge.net

[48] McCormick SF and Wade JG, 1993, Multigrid solution of a linearized, regularized least-squares problem in electrical impedance tomography, Inverse Problems, 9, 697-713.

[49] Meng S, West R, Ackroyd R, 2004, Markov Chain Monte Carlo techniques and spatial-temporal, modelling for medical EIT, this issue.

[50] Metherall P, Barber DC, Smallwood RH and Brown BH, 1996, Three Dimensional Electrical Impedance Tomography, Nature 380, 509-512.

[51] Metherall P, 1998, Three Dimensional Electrical Impedance Tomography of the Human Thorax, $\mathrm{PhD}$ thesis, University of Sheffield.

[52] Molinari M, 2003, High Fidelity Imaging in Electrical Impedance Tomography, PhD thesis, University of Southampton.

[53] Natterer F, 1982, The Mathematics of Comuterized Tomogrpahy, Wiley, Chichester.

[54] Patterson RP, Zhang J, 2003, Evaluation of an EIT reconstruction algorithm using finite difference human thorax models as phantoms, Physiol Meas,24, 467-475.

[55] Polydorides N and Lionheart WRB, 2002, A Matlab toolkit for three-dimensional electrical impedance tomography: a contribution to the Electrical Impedance and Diffuse Optical Reconstruction Software project, Meas Sci Technol, 13, 1871-1883.

[56] Polydorides, N, 2002, Image Reconstruction Algorithms for Soft Field Tomography, PhD Thesis, UMIST.

[57] Polydorides N, Lionheart WRB, McCann H, 2002, Krylov subspace iterative techniques: on the detection of brain activity with electrical impedance tomography, IEEE Trans Med Imaging, $21,596-603$

[58] Saad Y and Schultz MH, 1986, GMRES: A generalized minimal residual algorithm for solving nonsymmetric linear systems, SIAM J. Sci. Statist. Comput., 7, 856-869.

[59] Santosa F, Vogelius M, 1991, A backprojection algorithm for electrical impedance imaging, SIAM J. Appl. Math., 50, 216-243

[60] Schappel B, 2003, Electrical Impedance Tomography of the Half Space: Locating Obstacles by Electrostatic Measurements on the boundary, Proceedings of the 3rd World Congress on Industrial Process Tomography, Banff, Canada, September 2-5, 788-793.

[61] Seppanen A, Vauhkonen M, Vauhkonen PJ, Somersalo E, and Kaipio JP ,2001, State estimation with fluid dynamical evolution models in process tomography an application to impedance tomography, Inverse Problems, 17, 467-484.

[62] Siltanen S, Mueller J, Isaacson D, 2000, An implementation of the reconstruction algorithms of Nachman for the 2D inverse conductivity problem, Inverse Problems, 16, 681-699.

[63] Somersalo E, Isaacson D and Cheney M 1992 A linearized inverse boundary value problem for Maxwell s equations J. Comput. Appl. Math., 42, 123-136.

[64] Soleimani M, Powell C, 2004, Black-box Algebraic Multigrid for the 3D Forward Problem arising in Electrical Resistance Tomography, this issue.

[65] Somersalo E, Cheney M and Isaacson D, 1992, Existence and uniqueness for electrode models for electric current computed tomography, SIAM J Appl Math, 52, 1023-1040.

[66] Tamburrino A, Rubinacci G, 2002, A new non-iterative inversion method in electrical resistance tomography, Inverse Problems, 18, 1809-1829 
[67] Tarantola A, 1987, Inverse Problem Theory, Elsevier.

[68] Vauhkonen M, Kaipio JP, Somersalo E and Karjalainen PA, 1997, Electrical impedance tomography with basis constraints, Inverse Problems, 13, 523-530 .

[69] Vauhkonen M, Karjalainen PA, and Kaipio JP, 1998, A Kalman Filter approach to track fast impedance changes in electrical impedancetomography, IEEE Trans Biomed Eng, 45, 486-493.

[70] Vauhkonen M, 1997, Electrical Impedance Tomography and Prior Information, PhD thesis, University of Kuopio.

[71] Vauhkonen PJ, Vauhkonen M, Savolainen T, Kaipio JP, 1998, Static three dimensional electrical impedance tomography, Proceedings of ICEBI'98, Barcelona, Spain, 411-414

[72] Vauhkonen M, Karjalainen PA, and Kaipio JP, 1998, A Kalman filter approach to track fast impedance changes in electrical impedance tomography, IEEE Trans Biomed Eng, 45, 486493.

[73] Vauhkonen M, Lionheart WRB, Heikkinen LM, Vauhkonen PJ and Kaipio JP, 2001 A Matlab package for the EIDORS project to reconstruct two-dimensional EIT images, Physiol Meas $22,107-11$

[74] Vauhkonen PJ, 1999, Second order and Infinite Elements in Three-Dimensional Electrical Impedance Tomography, Phil.Lic. thesis, Department of Applied Physics, University of Kuopio, Finland.

[75] Vauhkonen PJ, Vauhkonen M, Kaipio JP, 2000, Errors due to the truncation of the computational domain in static three-dimensional electrical impedance tomography, Physiol Meas, 21, 125-135.

[76] Vauhkonen PJ, Vauhkonen M, and Kaipio JP, 2001, Fixed-lag smoothing and state estimation in dynamic electrical impedance tomography, Int J Numer Methods Eng, 50, 2195-2209.

[77] Vogel C, 2001, Computational methods for inverse problems, SIAM, Philadelphia.

[78] Wade JG, Senior K and Seubert S, 2004, Convergence of Derivative Approximations in the Inverse Conductivity Problem, SIAM J. Appl. Math, to appear.

[79] Yang WQ, Spink DM, York TA and McCann H, 1999, An image reconstruction algorithm based on Landweber's iteration method for electrical capacitance tomography, Meas Sci Technol, 10, 1065-1069.

[80] York T (ed), 1999, Proceedings of the 1st World Congress on Industrial Process Tomography, Buxton, UK, (Leeds: VCIPT)

[81] Yorkey TJ, Comparing Reconstruction Methods for Electrical Impedance Tomography 1986, PhD Thesis, Department of Electrical and Computational Engineering, University of Wisconsin, Madison, Wisconsin.

[82] Zhang J, Mackie RL, Madden TR, 1995,3-D resistivity Forward modelling and Inversion using Conjugate Gradients, Geophysics, 60, 1313-1325.

[83] Zhu QS, McLeod CN, Denyer CW, Lidgey FJ and Lionheart WRB, 1994, Development of a real-time adaptive current tomograph Physiol. Meas, 15, A37-A43.

[84] Zlochiver S et al, 2004, Induced current EIT system - Experimental results and numerical simulations, this issue. 